\title{
Thalamo-cortical interactions modeled by weakly connected oscillators: could the brain use FM radio principles?
}

\author{
Frank C. Hoppensteadt, Eugene M. Izhikevich* \\ Center for Systems Science and Engineering, Arizona State University, Tempe, AZ 85287-7606, USA
}

\begin{abstract}
We consider all models of the thalamo-cortical system that satisfy the following two assumptions: (1) each cortical column is an autonomous oscillator; (2) connections between cortical columns and the thalamus are weak. Our goal is to deduce from these assumptions general principles of thalamo-cortical interactions that are independent of the equations describing the system. We find that the existence of synaptic connections between any two cortical columns does not guarantee that the columns interact: They interact only when there is a certain nearly resonant relation between their frequencies, which implies that the interactions are frequency modulated (FM). When the resonance relation holds, the cortical columns interact through phase modulations. Thus, communications between weakly connected cortical oscillators employ a principle similar to that in FM radio: The frequency of oscillation encodes the channel of communication, while the information is transmitted via phase modulations. If the thalamic input has an appropriate frequency, then it can dynamically link any two cortical columns, even those that have non-resonant frequencies and would otherwise be unlinked. Thus, by adjusting its temporal activity, the thalamus has control over information processing taking place in the cortex. Our results suggest that the mean firing rate (frequency) of periodically spiking neuron does not carry any information other than identifying a channel of communication. Information (i.e. neural code) is carried through modulations of interspike intervals. (C) 1998 Elsevier Science Ireland Ltd. All rights reserved.
\end{abstract}

Keywords: Quasiperiodic oscillators; Canonical model; FM interactions; Neural code; Hodgkin-Huxley; WilsonCowan

\section{Introduction}

\subsection{The nature of neural code}

* Corresponding author.

E-mail: Eugene.Izhikevich@asu.edu;

URL: http://math.la.asu.edu/ eugene
One of the most intriguing questions in neuroscience is the nature of the neural code: What does column (neuron) 1 tell column (neuron) 2 in 
Fig. 1? Is information encoded in the mean firing rate, in the interspike intervals, or in something else? (See Fujii et al. (1996) for detailed discussion of this issue.)

In the present paper we address a simpler question: Do columns 1 and 2 interact? We say that they interact when rhythmic activity of one of them is sensitive to changes in the rhythmic activity of the other one. For example, when the columns are disconnected, they do not interact. Surprisingly, the existence of synaptic connections between two weakly connected columns having rhythmic activity does not guarantee interaction. To interact they should establish certain resonant relation between their frequencies. This fact was proven by Hoppensteadt and Izhikevich (1997) for the case of periodic rhythmic activity and later generalized by Izhikevich (1998) for the case of quasiperiodic (multi-frequency) rhythmic activity. We do not present the proofs here, since they involve normally hyperbolic invariant manifold, averaging, and KAM (Kolmogorov-ArnoldMoser) theories. Instead, we present here a detailed discussion of our assumptions and illustrate some possible neurobiological consequences.

\subsection{The canonical model approach}

Mathematical models in neuroscience have restricted value since the results can depend on particulars of the models. Various models of the same brain structure could produce various results. In contrast, our goal is to derive results that are largely independent of the model and that can be observed in a broad class or a family of neural models. To carry out this task we reduce the entire family of models to a canonical model (Hoppensteadt and Izhikevich 1997). Briefly, a model is canonical for the family if every model from the family can be transformed into this one by a continuous change of variables. The change of variables is usually not invertible, so we lose something in return for generality.

It is a difficult task to derive a canonical model for a broad family of neural models. Some partial success is achieved when the family describes neurons, columns or brain structures operating in a critical regime, such as near a bifurcation (Hoppensteadt and Izhikevich 1997). Then, the question of biological plausibility of a canonical model is replaced by the question of plausibility of a critical regime. This is a clear advantage of using canonical models.

\subsection{Major Assumptions}

In this paper we study a broad family of models that describe the thalamo-cortical system. We require that each model from the family satisfies two assumptions:

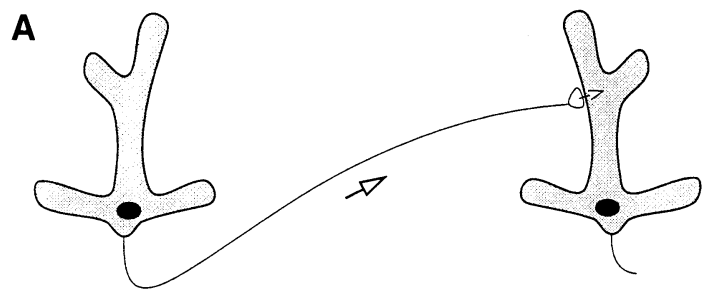

Neuron 1

Neuron 2
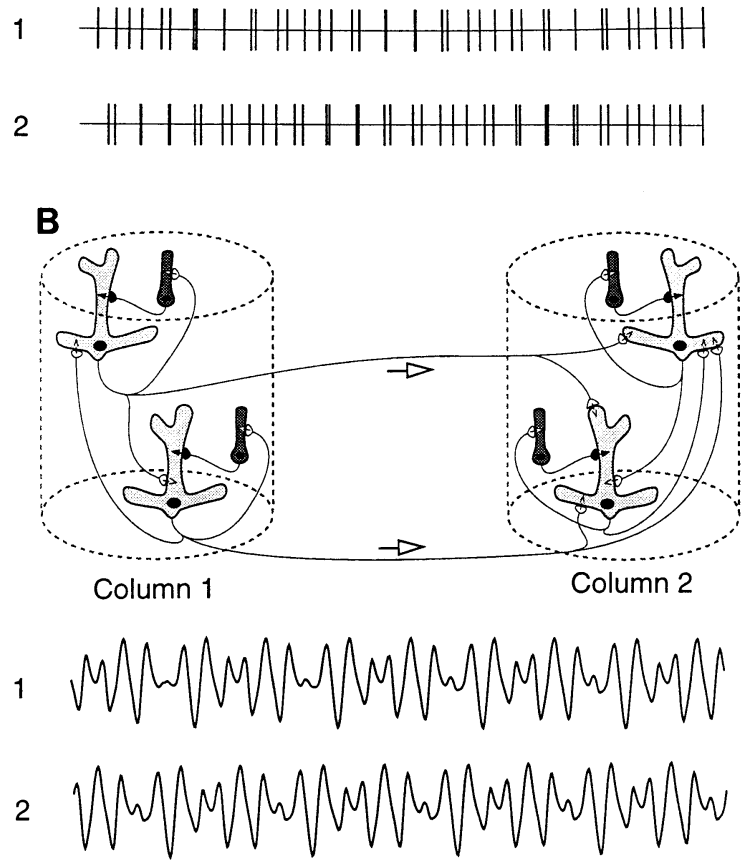

Fig. 1. The nature of neural code for neurons (A) and columns (B): What does neuron (column) 1 tell neuron (column) 2? We study a simpler question: Do neurons (columns) 1 and 2 interact? (from Izhikevich, 1998). 
- the thalamus and each cortical column is an endogenous (autonomous) oscillator.

- The oscillators are weakly connected.

We discuss these assumptions in detail in Section 2 .

\subsection{Major results}

In Section 3.1 we prove that any mathematical model satisfying these assumptions can be transformed into a phase model by a continuous noninvertible change of variables. Therefore, the phase model is canonical.

In Section 3.2 we show that if two cortical oscillators have non-resonant frequencies, then the corresponding phase variables do not depend on each other; that is, they do not interact. This implies that the existence of synaptic connections between any two cortical oscillators does not guarantee that the oscillators interact; but they can interact only when there is a certain (resonance) relation between their frequencies. Therefore, the interactions are frequency modulated (FM). When such a relation holds, the cortical oscillators interact through phase deviations, which are also referred to as being frequency modulations. Thus, communication between such weakly connected cortical oscillators employs FM radio principle: the frequency of oscillation encodes the channel for communication, and the information is transmitted via phase modulations.

From our analysis it follows that the cortical network can be partitioned by frequencies into relatively independent ensembles of columns processing information without cross-interference. Each column can change its membership between ensembles simply by adjusting its frequency of oscillations.

We also show that if the thalamic input has an appropriate frequency, then it can dynamically link any two cortical oscillators that would be unlinked otherwise. Thus, by adjusting its temporal activity, the thalamus has control over information processing taking place between cortical columns.

In Section 4.2 we illustrate the result using two weakly connected space clamped Hodgkin-Huxley oscillators. Our theory leads to the following two conclusions:
- the mean firing rate of a periodically spiking neuron does not carry any information other than identifying a channel of communication.

- information (neural code) is carried through modulations of interspike intervals.

The latter have been expressed many times before in the context of non-oscillating neurons (see, e.g. Abeles, 1982; Abeles et al., 1994; Fujii et al., 1996 for extensive review), while the former is somewhat novel. In Section 4.3 we use the WilsonCowan model to illustrate how the theory can be applied to quasiperiodic (multi-frequency) oscillators.

We stress that our usage of canonical models yields model-independent result. Thus, FM interaction is a genuine property of any mathematical model that satisfies only these assumptions.

\section{Assumptions}

In this paper we establish results for a broad family of mathematical models satisfying the following two assumptions.

\subsection{Weak connections}

This assumption is discussed in detail by Hoppensteadt and Izhikevich (1997) and Izhikevich (1998). It is based on the observation that average amplitude of post synaptic potential is smaller than $1 \mathrm{mV}$, which implies that there must be many (a few hundred) presynaptic neurons firing simultaneously to make a given cell fire (McNaughton et al., 1981).

\subsection{Autonomous oscillations}

We assume that each cortical column can exhibit periodic activity that is not induced by a rhythmic input, but is an endogenous (intrinsic) dynamical property of the column. Such an activity can easily be observed in many neural models, e.g. in the Wilson-Cowan model of a neural oscillator (Wilson and Cowan 1973). Since the origin or brain rhythmic activity is far from being understood (Frégnac et al., 1994), it is not clear whether or not this assumption is satisfied in 


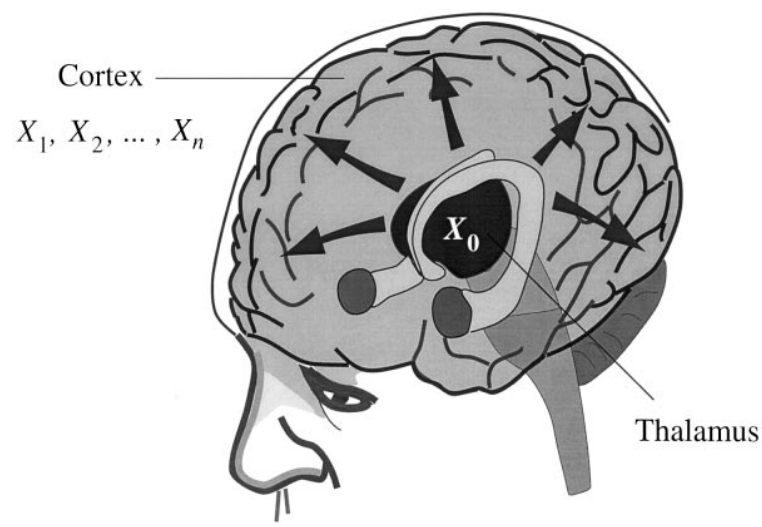

Fig. 2. System $(1,2)$ describes cortical oscillators forced by the thalamic input (from Izhikevich, 1998).

biological neural networks. Nonetheless, the assumption is certainly biologically plausible.

A cortical column may need a tonic (noisy) excitatory input to maintain oscillatory behavior. This does not contradict our assumption provided that the input is not rhythmic.

Finally, we notice that all our results can be extended for the case of quasiperiodic (multi-frequency) oscillations, such as those depicted in Figs. 1 and 6.

\section{Main theorems}

This is a technical section where we present our major mathematical results. Some readers may want to skip this section.

\subsection{The phase model}

Let the vector $X_{0} \in R^{m_{0}}$ describe activity of the thalamic network, and each vector $X_{i} \in R^{m}$ describe activity of the network comprising ith cortical column; see Fig. 2. Our approach is fairly general: We consider all possible mathematical models of the thalamo-cortical system that can be written in the form

$\dot{X}_{0}=F_{0}\left(X_{0}\right)+\varepsilon G_{0}\left(X_{0}, X_{1}, \ldots X_{n}\right)$

$\dot{X}_{i}=F_{i}\left(X_{i}\right)+\varepsilon \sum_{j=1}^{n} G_{i j}\left(X_{0}, X_{i}, X_{j}\right)$ where the unknown functions $F_{0}$ and $F_{i}$ describe dynamics of the thalamic network and the $i$ th cortical column, respectively; the unknown functions $G_{0}$ and $G_{i j}$ describe how the thalamus and the cortical columns interact. Notice that each $G_{i j}$ depends only on $X_{i}, X_{j}$ and $X_{0}$. A neurophysiological interpretation of this condition is discussed in detail by Izhikevich (1998).

The small parameter $\varepsilon \ll 1$ describes the strength of connections in the network. Hoppensteadt and Izhikevich, 1997 (Corollary 8.7) obtained the estimation

$0.004<\varepsilon<0.008$

for a model of the hippocampus using in vitro data (McNaughton et al., 1981).

Our assumption that the thalamus and each cortical column has periodic activity implies that each subsystem

$\dot{X}_{i}=F_{i}\left(X_{i}\right), \quad i=0, \ldots, n$,

has a limit cycle attractor, say $\gamma_{i}$, which is homeomorphic to the unit circle $S^{1}$. It is a well known fact (see, e.g. Malkin, 1949, 1956; Blechman, 1971; Neu, 1979; Ermentrout, 1981; Kuramoto, 1984; Ermentrout and Kopell, 1991) that the entire system $(1,2)$ can be approximated by a phase model that is defined on the $n+1$ dimensional torus $T^{n+1}$. Hoppensteadt and Izhikevich (1997) have gone further and proved that there is actually a continuous change of variables that transforms $(1,2)$ into the phase model. Below is the precise statement of this result.

Theorem 1 (phase equations for oscillatory neural networks) Consider a family of weakly connected systems of the form $(1,2)$ such that each equation in the uncoupled system $(\varepsilon=0)$

$\dot{X}_{i}=F_{i}\left(X_{i}\right), \quad i=0, \ldots, n$,

has an exponentially orbitally stable limit cycle attractor $\gamma_{i}$ having natural frequency $\boldsymbol{\Omega}_{i} \neq 0$. Then there is $\varepsilon_{0}>0$ and an open neighborhood $W$ of $M=\gamma_{0} \times \gamma_{1} \times \ldots \times \gamma_{n} \subset R_{0}^{\text {mt nm }}$ such that for all $0<\varepsilon \leq \varepsilon_{0}$ there is a continuous mapping $p: W \times$ $\left[0, \varepsilon_{0}\right] \rightarrow T^{n+1}$ that transforms solutions of $(1,2)$ to those of the phase model 
$\dot{\theta}_{0}=\Omega_{0}+\varepsilon h_{0}\left(\theta_{0}, \theta_{1}, \ldots, \theta_{n}, \varepsilon\right)$

$\dot{\theta}_{i}=\Omega_{i}+\varepsilon \sum_{j=1}^{n} h_{i j}\left(\theta_{0}, \theta_{i}, \theta_{j}, \varepsilon\right)$

where each $\theta_{i} \in S^{1}$ is the phase of oscillation of the ith cortical column, $i=1, \ldots, n$.

The proof of the theorem involves several steps, which we outline below. First we show (Lemma 4.5 in Hoppensteadt and Izhikevich, 1997) that the direct product of limit cycles, $M=\gamma_{0} \times \gamma_{1} \times$ $\ldots \times \gamma_{n}$, is a normally hyperbolic invariant manifold for the uncoupled $(\varepsilon=0)$ system $(1,2)$. Then we show (Theorem 4.7 in Hoppensteadt and Izhikevich, 1997) that a restriction of $(1,2)$ onto $M$ is a local model for all $\varepsilon \leq \varepsilon_{0}$; that is, there is an open neighborhood $W$ of $M$ and a (non-invertible) mapping $p_{W}: W \rightarrow M$ that projects each solution of $(1,2)$ in $W$ to a solution on $M$. Finally, we use an invertible change of variables $q: M \rightarrow \mathbb{T}^{n+1}$ to convert $(1,2)$ on $M$ into the phase model above. The mapping $p$ mentioned in the theorem is the composition of $p_{W}$ and $q$.

\subsection{Frequency modulated interactions}

Even though the phase model $(3,4)$ is much simpler than any system of the form $(1,2)$, its analysis still poses a problem since we do not know the form of the connection functions $h_{0}$ and $h_{i j}$. Thus, we cannot answer the question 'What can the phase model do?' Nevertheless, we may use the theorem below to answer the question 'What is it that the phase model $(3,4)$ cannot do regardless of the form of the functions $h_{0}$ and $h_{i j}$ ?' Resonance relations among the frequencies play a crucial role here.

We say that a vector of frequencies, $\Omega \in R^{n}$, is resonant if there is a nonzero integer vector $k \in Z^{n}$ such that

$k \cdot \Omega=\sum_{j=1}^{n} k_{i} \Omega_{i}=0$.

The vector is nonresonant when $k \cdot \Omega \neq 0$ for all nonzero $k \in Z^{n}$. When $n=2$, the vector $\boldsymbol{\Omega}=$ $\left(\Omega_{1}, \Omega_{2}\right)$ is resonant when $\Omega_{1}$ and $\Omega_{2}$ are commensurable; that is, when the ratio $\Omega_{1} / \Omega_{2}$ is a rational number; see detailed discussion by Hoppensteadt and Izhikevich (1997), Chapter 9).

Theorem 2 There is a near identity change of variables

$\theta_{i}(t)=\vartheta_{i}(t)+o(\varepsilon t), \quad i=0, \ldots, n$,

that transforms (Eq. (4)) into the form

$\dot{\vartheta}_{i}=\Omega_{i}+\varepsilon \sum_{j=1}^{n} H_{i j}\left(\vartheta_{0}, \vartheta_{i}, \vartheta_{j}\right)$

having the following important property: If the concatenated frequency vector $\left(\Omega_{0}, \Omega_{i}, \Omega_{j}\right) \in R^{3}$ is nonresonant, then the corresponding function $H_{i j}$ is a constant.

The proof follows from an obvious modification of the proof of Theorem 9.12 by Hoppensteadt and Izhikevich (1997). The theorem also follows from a similar theorem for quasiperiodic oscillators (Izhikevich, 1998).

Corollary 3 If the concatenated frequency vector $\left(\Omega_{0}, \Omega_{i}, \Omega_{j}\right) \in R^{3}$ is nonresonant, then the $i$ th and the $j$ th cortical oscillators do not interact on the large time scale of order $1 / \varepsilon$.

Indeed, it follows from the theorem that $H_{i j}=$ const in this case, which implies that $\vartheta_{j}$ does not participate in the $i$ th phase equation.

Corollary 4 If the frequencies $\Omega_{i}$ and $\Omega_{j}$ are commensurable, then the $i$ th and the $j$ th cortical oscillators may interact on a large time scale of order $1 / \varepsilon$ regardless of the input from the thalamus.

Since $\Omega_{i} / \Omega_{j}=k_{i} / k_{j}$ for some positive integers $k_{i}, k_{j} \in \mathbb{Z}$, the concatenated frequency vector $\left(\Omega_{0}, \Omega_{i}, \Omega_{j}\right)$ has a resonant relation corresponding to $k=\left(0, k_{j},-k_{i}\right) \in \mathbb{Z}^{3}$.

Corollary 5 If the frequencies $\Omega_{i}$ and $\Omega_{j}$ are incommensurable, then the $i$ th and the $j$ th cortical oscillators may interact only when the thalamic input has frequency $\Omega_{0}$ that is resonant with the pair $\left(\Omega_{i}, \Omega_{j}\right)$.

The simplest case when this happens is when

$\Omega_{0}=\left|\Omega_{i} \pm \Omega_{j}\right|$. 
We see that in order to interact, the cortical oscillators must establish certain resonance relation between their frequencies. In analogy with the FM radio, we say that the interactions are frequency modulated (FM). We discuss neurophysiological consequences of this result in Section 5.

\section{Examples}

An apparent advantage of our canonical model approach is that we derived universal results that do not depend on the particulars of equations describing the dynamics of cortical columns and the thalamus. Moreover, the same results would be obtained if each unit $\dot{X}_{i}=F_{i}\left(X_{i}\right)$ described dynamics of a dendritic spine, a single neuron, or an entire brain structure. As soon as each such unit exhibits autonomous rhythmic activity, and the units are weakly connected, they display FM interactions.

First, we illustrate a number of important issues using space clamped Hodgkin-Huxley equations to model two weakly connected spiking neurons. Then, we illustrate the quasiperiodic case using the Wilson-Cowan equations to model weakly connected cortical oscillators having multi-frequency rhythmic activity.

\subsubsection{Phase perturbation test}

One cannot determine whether or not two oscillators interact by examining their solution curves. Indeed, it is not apparent which features of the curves at the bottom of Fig. 1 are due to the autonomous oscillatory properties of the cortical columns and which are due to the weak interactions. To reveal the interactions, we employ the following phase perturbation test (Izhikevich 1998): We perform two simulations with the same parameters and initial conditions, but during the second simulation we apply a brief strong stimulus to the first neuron or column to change its phase. The new (perturbed) solutions are plotted as dashed curves in Figs. 4 and 6. If the second neuron or column 'feels' the perturbation, then its dashed solution would differ from the continuous one. This would imply that the neurons (columns) interact.

\subsection{Hodgkin-Huxley oscillators}

We simulated two weakly connected periodically spiking space clamped Hodgkin-Huxley neurons (Hodgkin and Huxley 1952). The phase of oscillation of a periodically spiking neuron determines the timing of its spikes; see Fig. 3.

Since the averaged PSP amplitude is small (of order $\varepsilon$ ), a single firing of the presynaptic neuron makes only small changes in the membrane potential of the postsynaptic one. Such small changes cannot stop an oscillation or change significantly its amplitude or frequency. They can only produce a tiny advance or delay in the next firing of the postsynaptic neuron. Such an advance or delay is called a phase shift; see Fig. 3. Even though the phase shift is negligible on a time scale of one period, it may become substantial on a longer time scale of order $1 / \varepsilon$, since small postsynaptic phase shifts may accumulate with each cycle. This is a typical example of temporal integration.

When the neurons have equal frequencies, they usually lock; see continuous curves in the upper part of Fig. 4. To find out whether the phase of the postsynaptic oscillator is sensitive to that of the presynaptic one, we use the phase perturbation test described above: We repeat our simulation (dashed curves) with the same parameters and initial conditions, but apply a strong brief impulse of current to the presynaptic oscillator to change its phase. It is easy to see that the postsynaptic oscillator is phase shifted too. Thus, the change in the presynaptic phase induces a corre-

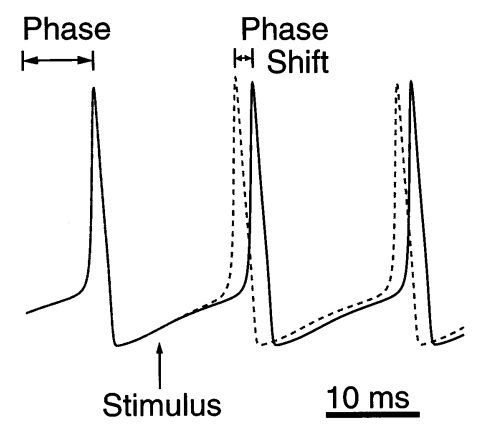

Fig. 3. Phase of oscillations in Hodgkin-Huxley model can be treated as timing of spikes. Weak synaptic input introduces small phase shift (dashed curve). 
A

Phase Resetting Stimulus

1

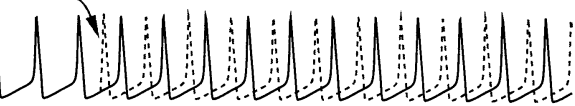

2

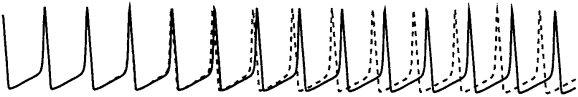

B

Phase Resetting Stimulus

$\underline{20 \mathrm{~ms}}$

1

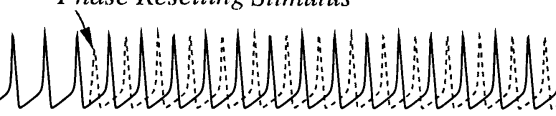

2

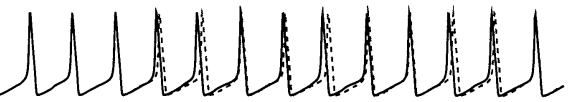

Fig. 4. Temporal integration of a periodic input in neurons (see Fig. 1A) depends on the frequency of the input. A: Neurons have identical frequencies. If a brief strong stimulus is applied to neuron 1 to change its phase (dashed curve), neuron 2 can 'feel' the changes by acquiring a phase shift. B: Neurons have different frequencies. The postsynaptic neuron is relatively insensitive to the phase of the presynaptic one (no significant phase shift). (All are simulations of space-clamped Hodgkin-Huxley equations).

sponding change in the postsynaptic phase, which can be interpreted as a transfer of information.

Now consider the case of different frequencies depicted in the lower part of Fig. 4. Even though we changed only the frequency of the presynaptic firing (all other parameters and initial conditions are unchanged), the postsynaptic neuron becomes relatively insensitive to the phase of the input - it does not acquire a phase shift.

Due to the specific nature of Hodgkin-Huxley model, the phenomenon depicted in Fig. 4 may be explained as follows: Each presynaptic spike induces either phase delay or phase advance depending on the time of its arrival. If it arrives immediately before firing of the postsynaptic cell, then it advances slightly the firing (i.e. phase advance). In contrast, its arrival immediately after the postsynaptic firing delays the next firing of the postsynaptic cell (i.e. phase delay). In the upper part of Fig. 4, the presynaptic phase is changed in such a way that each presynaptic firing occurs before the postsynaptic one. This produces slight phase advances, which accumulate and become significant. When the neurons have different frequencies, as in the lower part of Fig. 4, then presynaptic firings occur before, during, and after postsynaptic firings, which produces phase advances and delays in a seemingly random order. Such phase shifts do not accumulate but cancel each other on average.

The numerical experiment above corroborates the canonical model's prediction that the postsynaptic phase may or may not depend on the phase of presynaptic oscillations, depending on the relationship between pre and postsynaptic frequencies. When the frequencies are nearly identical, the phase of the presynaptic oscillation affects the postsynaptic phase through phase locking. When the frequencies are essentially different (see below), there are no significant shifts in the postsynaptic phase even on a long time scale of order of hundreds of milliseconds.

To quantify how the presynaptic phase affects the postsynaptic one, we change gradually the former and measure the greatest phase shift acquired by the latter. Performing such a procedure for various $\varepsilon$ and various pre and postsynaptic frequency ratios yields Fig. 5. The black regions, which cover the Arnold tongues, denote the areas where the postsynaptic neuron acquires a sizable phase shift due to the phase of the presynaptic one. The fact that black regions are mingled with the light ones for any fixed small $\varepsilon$ implies that interactions are frequency modulated (FM). Notice also that in order to allow interactions, the frequency ratio does not have to be rational: It must be in an $\varepsilon$-neighborhood of $p: q$ for some small integers $p$ and $q$. From Fig. 5 we see that interactions between weakly connected HodgkinHuxley oscillators are frequency modulated even for intermediate values of $\varepsilon$.

\subsection{Wilson-Cowan oscillators}

Now we consider two weakly connected cortical columns having quasiperiodic rhythmic activity. The easiest way to get such activity is to model each column by two strongly connected WilsonCowan neural oscillators; see top of Fig. 6. The equations and parameters for our simulations can be found in Izhikevich (1998).

Since activity of each column is quasiperiodic, its frequency is not a number, but a vector $\Omega_{i} \in R^{k}$. 
This results in a discrete power spectrum having many peaks corresponding to various components of the frequency vector $\Omega_{i}$ and their linear combinations; see top of Fig. 6. It has been proven (Izhikevich, 1998) that the columns interact only when the concatenated frequency vector $\left(\Omega_{i}, \Omega_{j}\right) \in R^{2 k}$ is resonant. When the columns are identical, they have resonant frequency vectors, and the phase perturbation test (middle of Fig. 6) confirms that the columns interact. In contrast, when their frequencies are mutually non-resonant, the columns do not interact, as one can see at the bottom of Fig. 6 .

\section{Discussion}

In this paper we consider all possible mathematical models of the thalamo-cortical system having two features:

1. each cortical column is an autonomous oscillator.

2. cortical oscillators and the thalamus are weakly connected.

Instead of studying all such models, we prove that each of them can be transformed into the canonical model $(3,4)$ by a continuous non-invertible

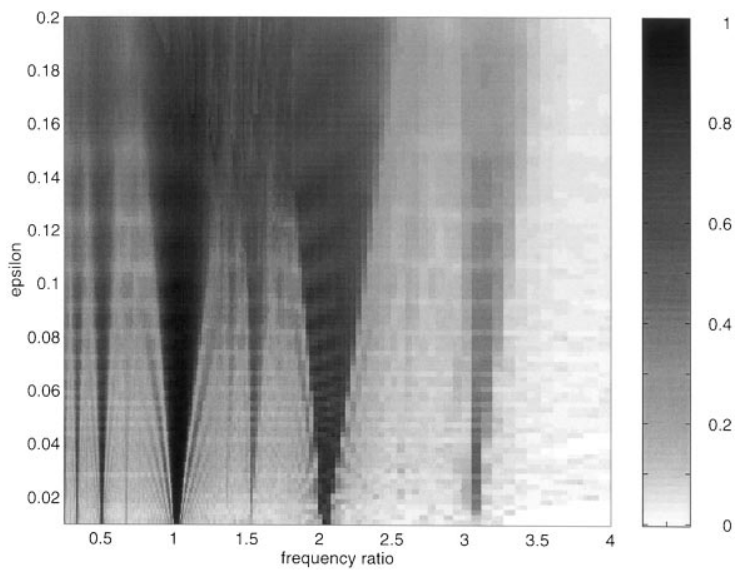

Fig. 5. Sensitivity of a Hodgkin-Huxley oscillator to a weak periodic input on the time scale $[0,10 / \varepsilon]$ is plotted as a function of the strength of connections $\varepsilon$ and the frequency ratio (the darker the dot, the more sensitive the oscillator is). We see that there are FM interactions even for intermediate $\varepsilon$ (notice the Arnold tongue structure).

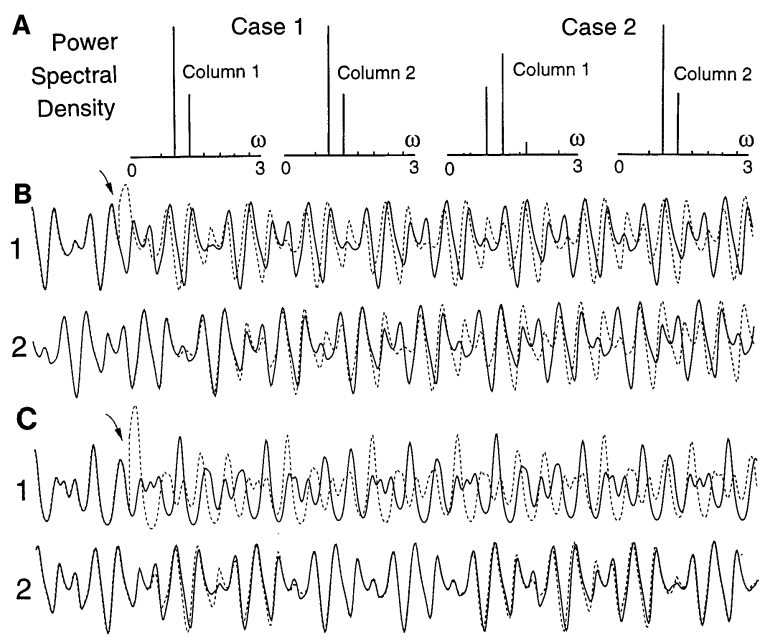

Fig. 6. A: Frequency modulated (FM) interactions: Interactions between two cortical columns (see Fig. 1B) exhibiting quasiperiodic activity depend on their frequency vectors (A). B: Identical frequency vectors. If a brief strong stimulus is applied to column 1 to change its phase (dashed curve), column 2 acquires a phase shift too. Therefore, the columns interact through phases. C: Mutually nonresonant frequency vectors. Column 2 is relatively insensitive to any changes of phase of column 1. Therefore, the columns do not interact, even though they are connected (from Izhikevich, 1998).

change of variables. The advantage of this canonical model approach is that the results are modelindependent. Many more canonical models for neuroscience applications are derived by Hoppensteadt and Izhikevich (1997).

We still do not know how the thalamo-cortical system functions and what it can accomplish from the neurocomputational point of view. Nevertheless, we know what it cannot accomplish regardless of the equations describing its dynamics. When the cortical oscillators have nearly incommensurable frequencies and there is no thalamic forcing, any interactions between such cortical oscillators are functionally insignificant (i.e. they average to a constant) even when there are synaptic connections between them. When the cortical oscillators have nicely commensurable frequencies, they interact via phase deviations (frequency modulations, FM). In this sense weakly connected cortical oscillators employ FM radio principle: Frequency encodes the channel of communication, while phase modulations encode the information to be transmitted via the channel. 
Our theory has the following implication: An entire cortex can be dynamically partitioned into interwoven, but relatively independent, ensembles that process information without cross-interference. A cortical oscillator may participate in different ensembles by changing its frequency, without changing the strengths of synaptic connections. When the column has a quasiperiodic (multi-frequency) activity, as in Fig. 6, it may participate in different ensembles simultaneously (Izhikevich, 1998). To find out what kind of information processing takes place inside such ensembles, one should study the canonical model (2). Hoppensteadt and Izhikevich (1997), Theorem 9.15) proved that the phase model may have oscillatory associative memory such that each memorized pattern is represented by a limit cycle attractor.

The functional partition of the cortex may be dynamically reorganized by a rhythmic input from the thalamus. Indeed, the thalamus can link any 'non-interacting' cortical columns if it has an appropriate frequency (Corollary 5). When there is a need to link many cortical oscillators, the thalamic input must be quasiperiodic or chaotic having appropriate frequencies in its power spectrum.

Since our approach is model-independent, it can also be applied to the hippocampus forced by the septum, to the olfactory cortex forced by the olfactory bulb, or to any other brain system that satisfies the assumptions discussed in Section 2. Verifying the assumptions is essential, since we can easily present counterexamples showing that if either of them is violated, the FM interactions might not take place.

One of the consequences of our usage of the canonical model approach is that we can apply our theory to weakly connected spiking neurons. Our result that interactions between periodically spiking cells are frequency modulated agrees with the experimental finding of effective connectivity in the mammalian cortex (Aertsen and Gerstein, 1991). The result that information is transmitted via phase modulations, which are referred to as being frequency modulations in electrical engineering literature or modulations of interspike intervals in neuroscience literature, resembles the theory of syn-fire chains (Abeles, 1991; Abeles et al., 1994). The result that the neural network can be subdivided into relatively independent ensembles, which can be reorganized by an external input, resembles the theory of a dynamical cell assembly proposed by Fujii et al. (1996), who refer to the external input as being a context.

Finally, we contrast our theory with the theory of dynamic link architecture proposed by von der Malsburg (1995); (see also Terman and Wang, 1995), which considers neurons having identical frequencies but different phases. It postulates that in order to communicate the neurons have to be in-phase synchronized. Thus, the phase of firing encodes the channel of communication, whereas the frequency is an unused parameter in the dynamic link architecture theory. That theory and ours do not contradict, but rather complement each other.

\section{References}

Abeles, M., 1982. Role of the cortical neuron: integrator or coincidence detector. Isr. J. Med. Sci. 18, 83-92.

Abeles, M., 1991. Corticonics: Neural Circuits of The Cerebral Cortex. Cambridge University Press, New York.

Abeles, M., Prut, Y., Bergman, H., Vaadia, E., 1994. Synchronization in neuronal transmission and its importance for information processing. In: Buzsáki, G., Llinás, R., Singer, W., Berthoz, A., Christen, Y. (Eds.), Temporal Coding in the Brain. Springer-Verlag, New York.

Aertsen, A.M.H.J., Gerstein, G.L., 1991, Dynamic aspects of neuronal cooperativity: fast stimulus-locked modulations of effective connectivity. In: Kruger J. (Ed.), Neuronal Cooperativity. SpringerVerlag, Berlin.

Blechman, I.I., 1971. Sinchronizatzia Dinamicheskich Sistem (Synchronization of Dynamical Systems) Science, Moscow.

Frégnac, Y., Bringuier, V., Baranyi, A., 1994. Oscillatory neuronal activity in visual cortex: a critical re-evaluation. In: Buzsáki et al. (Eds.), Temporal Coding in the Brain. Springer-Verlag, New York.

Fujii, H., Ito, H., Aihara, K., Ichinose, N., Tsukada, M., 1996. Dynamical cell assembly hypothesis; theoretical possibility of spatio-temporal coding in the cortex. Neural Netw 9, $1303-1350$.

Ermentrout, G.B., 1981. n:m Phase-locking of weakly coupled oscillators. J. Math. Biol. 12, 327-342.

Ermentrout, G.B., Kopell, N., 1991. Multiple pulse interactions and averaging in systems of coupled neural oscillators. J. Math. Biol. 29, 195-217. 
Hodgkin, A.L., Huxley, A.F., 1952. A quantitative description of membrane current and application to conduction and excitation in nerve. J. Physiol. 117, 500-544.

Hoppensteadt, F.C., Izhikevich, E.M., 1997. Weakly Connected Neural Networks. Springer-Verlag, New York.

Izhikevich, E.M., 1998. Weakly connected quasiperiodic oscillators; FM interactions; and multiplexing in the brain, SIAM J. Appl. Math. (Accepted).

Kuramoto, Y., 1984. Chemical Oscillations, Waves, and Turbulence. Springer-Verlag, New York.

Malkin, I.G., 1949. Metodi Puankare i Liapunova v teorii nelineinix kolebanii (Methods of Poincare and Liapunov in theory of non-linear oscillations). Gostexizdat, Moscow.

Malkin, I.G., 1956. Nekotorye zadachi teorii nelineinix kolebanii (Some problems in nonlinear oscillation theory). Gostexizdat, Moscow. von der Malsburg, C., 1995. Dynamic link architecture. In: Arbib, M.A., (Ed.), Brain Theory and Neural Networks. MIT press, Cambridge, MA.

McNaughton, B.L., Barnes, C.A., Andersen, P., 1981. Synaptic efficacy and EPSP summation in granule cells of rat fascia dentata studied in vitro. J. Neurophysiol. 46, 952966.

Neu, J.C., 1979. Coupled chemical oscillators. SIAM J. Appl. Math. 37, 307-315.

Terman, D., Wang, D., 1995. Global competition and local cooperation in a network of neural oscillators. Physica D $81,148-176$.

Wilson, H.R., Cowan, J.D., 1973. A mathematical theory of the functional dynamics of cortical and thalamic nervous tissue. Kybernetik 13, 55-80. 